\title{
Dynamic crossover in the global persistence at criticality
}

\author{
Raja Paul, ${ }^{1}$ Andrea Gambassi, ${ }^{2,3}$ and Grégory Schehr ${ }^{4}$ \\ ${ }^{1}$ BIOMS, IWR, University of Heidelberg, 69120 Heidelberg, Germany \\ ${ }^{2}$ Max-Planck-Institut für Metallforschung, Heisenbergstr. 3, 70569 Stuttgart, Germany \\ ${ }^{3}$ Institut für Theoretische und Angewandte Physik, \\ Universität Stuttgart, Pfaffenwaldring 57, 70569 Stuttgart, Germany \\ ${ }^{4}$ Laboratoire de Physique Théorique (UMR du CNRS 8627), \\ Université de Paris-Sud, 91405 Orsay Cedex, France
}

\begin{abstract}
We investigate the global persistence properties of critical systems relaxing from an initial state with non-vanishing value of the order parameter (e.g., the magnetization in the Ising model). The persistence probability of the global order parameter displays two consecutive regimes in which it decays algebraically in time with two distinct universal exponents. The associated crossover is controlled by the initial value $m_{0}$ of the order parameter and the typical time at which it occurs diverges as $m_{0}$ vanishes. Monte-Carlo simulations of the two-dimensional Ising model with Glauber dynamics display clearly this crossover. The measured exponent of the ultimate algebraic decay is in rather good agreement with our theoretical predictions for the Ising universality class.
\end{abstract}

PACS numbers: 05.70.Jk, 05.50.+q

\section{INTRODUCTION}

In spite of many efforts during the last decades, a detailed description of the non-equilibrium dynamics of statistical systems is still lacking. In addition to disordered and glassy systems [1] even simpler ones (e.g., pure magnets) have revealed unexpected non-equilibrium dynamical behaviors such as aging [2, 3]. Close to critical points, some aspects of these collective behaviors become largely independent of the microscopic details of the system (universality) and therefore they can be very effectively investigated by means of simplified models, among which fieldtheoretical (FT) [3] ones. Within this approach one studies, for instance, the non-equilibrium relaxation from a state with non-vanishing value $m_{0}$ of the time-dependent average order parameter $m(t)$ (e.g., the magnetization of a ferromagnet). It turns out [4] that, after a nonuniversal transient, $m(t)$ grows in time as $m(t) \propto m_{0} t^{\theta^{\prime}}$ for $t \ll \tau_{m} \propto m_{0}^{-1 / \kappa}$ whereas, for $t \gg \tau_{m}, m(t)$ decays algebraically to zero as $m(t) \propto t^{-\beta /(\nu z)}$. These different time dependences are characterized by the universal exponents $\theta^{\prime}$ (the so-called initial-slip exponent [4]) and $\kappa=\theta^{\prime}+\beta /(\nu z)$, where $\beta, \nu$ and $z$ are the usual static and dynamic (equilibrium) critical exponents, respectively. In addition to the actual time dependence of $m(t)$, the persistence properties of thermal fluctuations $\delta m(t)$ around $m(t)$ provide useful informations on the dynamics of the system (especially on its non-Markovian nature) and indeed in recent years they have attracted, in various contexts, considerable attention both theoretically [5] and experimentally [6]. For a stochastic process $\{X(t)\}_{t \geq 0}$ with zero mean, the persistence probability $P(t)$ is defined as the probability that $X(t)$ does not change sign up to time $t$. A related quantity which might be used to characterize the process is the firstcrossing (or first-passage) time $t_{\text {cro }}$, defined as the time it takes $X(t)$ to change its sign for the first time. Clearly, the probability distribution $p\left(t_{\text {cro }}\right)$ is related to $P(t)$ via $p\left(t_{\text {cro }}\right)=-P^{\prime}\left(t_{\text {cro }}\right)$. For a ferromagnet at finite temperature $T$ the persistence probability of a single spin (i.e., of the fluctuations of the local order parameter) decays always exponentially in time due to fast thermal fluctuations. The long-time decay of the persistence probability $P_{c}(t)$ of $\delta m(t)$ (i.e., of the fluctuations of the global order parameter), instead, becomes algebraic upon approaching the critical point: $P_{c}(t) \propto t^{-\theta}$, with a non-trivial exponent $\theta>0$. This exponent was calculated analytically for some ferromagnetic models with different dynamics (Models A and C [7]) and random initial condition [8, [9] (i.e., $m_{0}=0$ ) and for reaction-diffusion systems in the directed percolation (DP) universality class, initially in the active phase [10] (i.e., $m_{0}=1$ on the lattice, where the order parameter is the spatial density of reacting particles). We shall denote $\theta_{0} \equiv \theta\left(m_{0}=0\right)$ and $\theta_{\infty} \equiv \theta\left(m_{0}=1\right)$. In the previous cases analytical progresses rely on the fact that the global fluctuating order parameter $m+\delta m$ has a Gaussian distribution for all finite times $t$ in the thermodynamic limit. Indeed, for a $d$-dimensional system of linear size $L, m+\delta m$ is given by the sum of $L^{d}$ random variables (the local fluctuating degrees of freedom, e.g., spins in ferromagnets) which are correlated only across a finite, time-dependent correlation length $\xi(t)$. In the thermodynamic limit $L / \xi(t) \gg 1$ the central limit theorem implies that $m+\delta m$ is a Gaussian process, for which powerful tools have been developed in order to determine the persistence exponent [5, 11]. Remarkably, under the additional assumption that the process is Markovian, $\theta$ can be related to known critical exponents, via two (hyper)scaling relations $\theta_{0} \equiv \mu_{0}=-\theta^{\prime}+(1-\eta / 2) / z$ (where $\eta$ is the Fisher exponent) and $\theta_{\infty} \equiv \mu_{\infty}=1+d /(2 z)$ which are valid for spin systems and for the DP universality class, respectively. For $m_{0}=0$ (weak) non-Markovian 
corrections were found at two-loop and one-loop order in a dimensional expansion around $d=4$, for Model A and Model C, respectively, in rather good agreement with Monte Carlo estimates $\theta_{0}=0.237(3)$ in dimension $d=2$ [12] and $\theta_{0} \simeq 0.41$ in $d=3$ [13]. For completely ordered initial conditions $\left(m_{0}=1\right)$, instead, these corrections have not been investigated so far beyond the case of DP, where they appear at one-loop order [10] and they were measured numerically in $d=1$ [14]. These results imply that the average and variance of the first-crossing time $t_{\text {cro }}$ are both finite for DP in $d<4$ but not for the spin models studied in Refs. [9, 11].

The evidence accumulated so far in two different models with $m_{0}=0$ and 1 show that the critical persistence properties actually depend on $m_{0}$. It is therefore natural to investigate how the corresponding crossover occurs when $m_{0}$ is varied within the same model [24] and which are the associated universal features.

\section{UNIVERSAL SCALING BEHAVIOR OF THE PERSISTENCE PROBABILITY}

Here we focus primarily on the Ising model (on a $d$ dimensional hypercubic lattice) with Glauber dynamics quenched to its critical point and we study its persistence properties both analytically and numerically. The universal aspects of the relaxation of this model are captured by the so-called Model A 7] for the $n$-component fluctuating local order parameter $\varphi(x, t)$ (e.g., the coarsegrained density of spins in the Ising model):

$$
\eta \partial_{t} \varphi(x, t)=-\frac{\delta \mathcal{H}[\varphi]}{\delta \varphi(x, t)}+\zeta(x, t)
$$

where $\zeta(x, t)$ is a Gaussian white noise with $\langle\zeta(x, t)\rangle=0$ and $\left\langle\zeta(x, t) \zeta\left(x^{\prime}, t^{\prime}\right)\right\rangle=2 \eta T \delta\left(x-x^{\prime}\right) \delta\left(t-t^{\prime}\right), \eta$ is the friction coefficient (set to 1 in the following) and $T$ the temperature of the thermal bath. In Eq. (1), $\mathcal{H}$ is the $O(n)$-symmetric Landau-Ginzburg functional which, for $n=1$, describes the universal aspects of the static properties of the Ising model:

$$
\mathcal{H}[\varphi]=\int \mathrm{d}^{d} x\left[\frac{1}{2}(\nabla \varphi)^{2}+\frac{1}{2} r_{0} \varphi^{2}+\frac{g_{0}}{4 !}\left(\varphi^{2}\right)^{2}\right]
$$

where $r_{0}$ is a parameter which has to be tuned to a critical value $r_{0, c}$ when approaching the critical temperature $T_{c}$ (here $r_{0, c}=0$ ) and $g_{0}>0$ is the bare coupling constant.

At the initial time $t=0$, the system is in a random configuration with mean magnetization $[\varphi(x, 0)]_{i}=M_{0}$ and short-range correlations $\left[\varphi(x, 0) \varphi\left(x^{\prime}, 0\right)\right]_{i}=\tau_{0}^{-1} \delta(x-$ $\left.x^{\prime}\right)\left([\ldots]_{i}\right.$ stands for the average over the distribution of the initial configuration), where $\tau_{0}^{-1}$ is irrelevant in determining the leading scaling properties [4] (therefore we set $\tau_{0}^{-1}=0$ ). For $n=1$ (the case $n>1$ is shortly discussed below), the stochastic process we are interested in is

$$
\psi(x, t)=\varphi(x, t)-M(t), \quad \text { where } \quad M(t)=\langle\varphi(x, t)\rangle
$$

is the average magnetization and $\langle\ldots\rangle$ stands for the average over the possible realizations of the stochastic noise $\zeta$. Defining $\tilde{M}(t)$ as

$$
\tilde{M}(t)=\frac{1}{L^{d}} \int \mathrm{d}^{d} x \psi(x, t)
$$

we are interested in the probability $P_{c}(t)$ that $\tilde{M}$ does not change sign in the time interval $[0, t]$ following the quench to $T_{c}$.

We first present the result of the Gaussian approximation which is exact in dimension $d>4$ and which is obtained by neglecting non-linear terms in $\psi$ in the Langevin Eq. (1) expressed in terms of $\psi(x, t)$ and $m^{2} \equiv$ $g_{0} M^{2} / 2$ (see, e.g., Ref. [15]):

$$
\begin{aligned}
& \partial_{t} \psi(x, t)=\left[\nabla^{2}-m^{2}(t)\right] \psi(x, t)+\zeta(x, t) \\
& \text { where } \quad \partial_{t} m(t)+\frac{1}{3} m^{3}(t)=0 .
\end{aligned}
$$

The equation of motion for $\tilde{M}$ [see Eq. (4)] readily follows: $\partial_{t} \tilde{M}(t)+m^{2}(t) \tilde{M}(t)=\tilde{\zeta}(t)$, where $\tilde{\zeta}(t)=$ $L^{-d} \int \mathrm{d}^{d} x \zeta(x, t)$ is still Gaussian and, from Eq. (5), $m^{2}(t)=\left(2 t / 3+m_{0}^{-2}\right)^{-1}$ where $m(t=0)=m_{0}$. In the limit $t \ll m_{0}^{-2}$, one finds that $\tilde{M}(t)$ executes a simple random walk and therefore $P_{c}(t) \propto t^{-1 / 2}[16$. In the opposite limit $t \gg m_{0}^{-2}, \tilde{M}(t)$ satisfies the Langevin equation $\partial_{t} \tilde{M}(t)+(3 / 2 t) \tilde{M}(t)=\tilde{\zeta}(t)$ which turns into $\partial_{\tau} \hat{M}(\tau)=\eta(\tau)$ when the variables $\hat{M}(t)=t^{3 / 2} \tilde{M}(t)$, $\eta(t)=t^{-3 / 2} \tilde{\zeta}(t) / 4$ and $\tau=t^{4}$ are introduced [8]. Accordingly, $P_{c}(\tau) \propto \tau^{-1 / 2}$, i.e., $P_{c}(t) \propto t^{-2}$, which decays to zero more rapidly than for $t \ll m_{0}^{-2}$ and clearly shows the existence of two different regimes. The full crossover function can be obtained by noticing that $\tilde{M}(t)$ is a Gaussian process and therefore it is completely determined by its two-time correlation function $C_{\tilde{M}}\left(t, t^{\prime}\right)=$ $\left\langle\tilde{M}(t) \tilde{M}\left(t^{\prime}\right)\right\rangle$ which can be easily calculated [15]. Following Ref. [8], one introduces the normalized process $X(t)=\tilde{M}(t) /\left\langle\tilde{M}^{2}(t)\right\rangle^{1 / 2}$, the two-time correlation function of which is given by $\left(t>t^{\prime}\right)$ [15]:

$$
\begin{aligned}
\left\langle X(t) X\left(t^{\prime}\right)\right\rangle & =\frac{C_{\tilde{M}}\left(t, t^{\prime}\right)}{\sqrt{C_{\tilde{M}}(t, t) C_{\tilde{M}}\left(t^{\prime}, t^{\prime}\right)}}=\frac{L\left(\tilde{t^{\prime}}\right)}{L(\tilde{t})} \\
\text { where } \quad L(\tilde{t}) & =\sqrt{(\tilde{t}+1)^{4}-1}
\end{aligned}
$$

and the dimensionless time variables are given by $\tilde{t}=$ $t / \tau_{m}$ with $\tau_{m}=3 /\left(2 m_{0}^{2}\right)$. In terms of the logarithmic time $T=\ln L(t), X(T)$ is a stationary Gaussian process with purely exponential correlations $\left\langle X(T) X\left(T^{\prime}\right)\right\rangle=$ $\exp \left(-\left|T-T^{\prime}\right|\right)$, for which the persistence probability is known exactly [5, 17]. In the limit $t, \tau_{m} \gg t_{\text {micr }}$ (where 
$t_{\text {micr }}$ is some non-universal microscopic time scale) one finds

$$
\begin{aligned}
P_{c}(t) \propto \frac{L\left(\tilde{t}_{\text {micr }}\right)}{L(\tilde{t})} & \simeq \frac{\left(\tau_{m} / t_{\text {micr }}\right)^{-1 / 2}}{\sqrt{\left(1+t / \tau_{m}\right)^{4}-1}} \\
& \sim \begin{cases}t^{-\frac{1}{2}}, & t \ll \tau_{m} \\
t^{-2}, & t \gg \tau_{m}\end{cases}
\end{aligned}
$$

in agreement with the results obtained by mapping the process on a random walk. The mean first-crossing time $t_{\text {cro }}$ resulting from $P_{c}(t)$ is finite for $m_{0} \neq 0$, whereas its variance is not. Although the Gaussian approximation is exact only for $d>4$, it clearly displays for finite $m_{0}$ (i.e., finite $\tau_{m}$ ) an interesting crossover (7) which is expected to occur for generic $d$ above the lower critical dimension of the model - between two successive regimes in which $P_{c}(t)$ decays algebraically with two different exponents, $\theta_{0}$ and $\theta_{\infty}$, respectively. The first regime $\left(t \ll \tau_{m}\right)$ has a temporal extent $\sim \tau_{m}$ which increases upon decreasing $m_{0}$ and indeed, in the limit $m_{0} \rightarrow 0$ considered in Refs. [8, 9], it is the only one accessible. The crossover to the second regime takes place for $t \simeq \tau_{m}$, with $\tau_{m}$ decreasing upon increasing $m_{0} \neq 0$ and indeed $\tau_{m}$ tends to zero in the case $m_{0} \rightarrow \infty$ considered in Ref. [10] for the DP.

To go beyond the Gaussian approximation, we take advantage of recent results about the aging behavior of systems described by Eqs. (1) and (2) following a quench from a state with $m_{0} \neq 0$ to the critical point [15]. As mentioned in the introduction, $\tilde{M}(t)$ (and therefore $\left.X(t)=\tilde{M}(t) /\left\langle\tilde{M}^{2}(t)\right\rangle^{1 / 2}\right)$ is a Gaussian process in the thermodynamic limit. Taking into account the scaling form of $C_{\tilde{M}}\left(t, t^{\prime}\right)$ discussed in Ref. [15], one expects for $t>t^{\prime}$ :

$$
\left\langle X(t) X\left(t^{\prime}\right)\right\rangle=\left(t / t^{\prime}\right)^{-\mu_{0}} F_{X}\left(t^{\prime} / t, t / \tau_{m}\right)
$$

where $\tau_{m} \equiv\left(B_{m} m_{0}\right)^{-1 / \kappa}, B_{m}$ is a non-universal constant which can be fixed according to some normalization condition (see Ref. [15] for details), $F_{X}$ is a universal scaling function (with $F_{X}(1, y)=1$ ) and $\mu_{0}=-\theta^{\prime}+(1-\eta / 2) / z$. For $t / \tau_{m} \ll 1$ one recovers the result of Refs. [8, 9]. However, a non-vanishing mean $m_{0}$ of the initial order parameter affects the behavior of the temporal correlations as soon as $t \sim \tau_{m}$ and in particular for $t, t^{\prime} \gg \tau_{m}$. The limiting behaviors of Eq. (8) can be derived from those of $C_{\tilde{M}}\left(t, t^{\prime}\right)$ discussed in Ref. [15]:

$$
\left\langle X(t) X\left(t^{\prime}\right)\right\rangle \sim \begin{cases}\left(t / t^{\prime}\right)^{-\mu_{0}} f_{0}\left(t^{\prime} / t\right), & t^{\prime}<t \ll \tau_{m} \\ \left(t / t^{\prime}\right)^{-\mu_{\infty}} f_{\infty}\left(t^{\prime} / t\right), & t>t^{\prime} \gg \tau_{m}\end{cases}
$$

where $f_{0}(x)=F_{X}(x, 0), f_{\infty}(x)=x^{\mu_{0}-\mu_{\infty}} F_{X}(x, y \rightarrow$ $\infty)$ with $f_{0, \infty}(0)$ finite $\left[f_{0, \infty}(1)=1\right]$ and, for $d<4$, $\mu_{\infty}=1+d /(2 z) 25$. Note that if $f_{0, \infty}(x)$ is constant for $x \in[0,1],\left\langle X(t) X\left(t^{\prime}\right)\right\rangle$ can be cast asymptotically in the form (6) with a suitable choice of $L(t)$ and therefore the process $X(t)$ can be mapped into a Markovian one with $P_{c}\left(t_{\text {micr }} \ll t \ll \tau_{m}\right) \sim t^{-\mu_{0}}$ and $P_{c}\left(t \gg \tau_{m}\right) \sim t^{-\mu_{\infty}}$. In the scaling limit, the relevant time scales for the problem of persistence are the time $t$ elapsed since the quench and the (mesoscopic) time scale $\tau_{m}$ set by the value of the initial magnetization. Accordingly, one expects (also on the basis of the Gaussian approximation) a scaling behavior

$$
P_{c}(t)=A_{P} t^{-\theta_{0}} \mathcal{P}\left(t / \tau_{m}\right), \quad \mathcal{P}(x \gg 1) \sim x^{-\theta_{\infty}+\theta_{0}}
$$

where $A_{P}$ is a non-universal constant such that $\mathcal{P}(0)=1$, $\mathcal{P}(x)$ is a universal scaling function, $\tau_{m}$ is fixed by the behavior of the magnetization as in Ref. [15], and $\theta_{\infty}$ is a yet undetermined exponent. Equation (10) implies $\left\langle t_{\text {cro }}\right\rangle=\int_{0}^{\infty} \mathrm{d} t P_{c}(t) \sim \tau_{m}^{1-\theta_{0}}$ and therefore the firstcrossing time $t_{\text {cro }}$ has a finite average for $\theta_{0}<1<\theta_{\infty}$ and a finite second moment $\left\langle t_{\text {cro }}^{2}\right\rangle=2 \int_{0}^{\infty} \mathrm{d} t t P_{c}(t) \sim \tau_{m}^{2-\theta_{0}}$ for $\theta_{0}<2<\theta_{\infty}$. Note that $\theta_{0, \infty}=\mu_{0, \infty}$ for a Markovian process. The corrections to $\theta_{0}$ due to non-Markovian effects (reflected in the fact that $f_{0, \infty}(x)$ in Eq. (9) are not constant) were obtained at two-loop order in Ref. [9]. For $\theta_{\infty}$ these corrections have not been calculated. They can be determined by focusing on the case $m_{0} \rightarrow \infty$ of Eq. (9), i.e., on $f_{\infty}(x)$, which was calculated in Ref. [15] up to one loop in the $\epsilon$-expansion $(\epsilon=4-d)$. Taking advantage of the results therein one finds, in logarithmic time $T=\ln t\left(T>T^{\prime}\right)$,

$$
\begin{aligned}
& \left\langle X(T) X\left(T^{\prime}\right)\right\rangle=\mathrm{e}^{-\mu_{\infty}\left(T-T^{\prime}\right)} \mathcal{A}\left(\mathrm{e}^{-\left(T-T^{\prime}\right)}\right), \\
& \text { where } \mathcal{A}(x)=1+\epsilon \mathcal{A}_{1}(x)+\mathcal{O}\left(\epsilon^{2}\right),
\end{aligned}
$$

$\mathcal{A}_{1}(x)=2\left[f_{C}(x)-f_{C}(1)\right] / 3$ and $f_{C}(x)$ is given in Eq. (B.31) of Ref. [15]. The universal function $\mathcal{A}_{1}(x)$ carries the signature of the non-Markovian corrections to $\theta_{\infty}$ which, in turn, can be calculated by using the perturbation theory of Ref. 9] (see Eq. (14) therein):

$$
\begin{aligned}
\mathcal{R} & \equiv \frac{\theta_{\infty}}{\mu_{\infty}}=1-\epsilon \frac{2 \mu_{\infty}}{\pi} \int_{0}^{1} \mathrm{~d} x \frac{x^{\mu_{\infty}-1} \mathcal{A}_{1}(x)}{\left(1-x^{2 \mu_{\infty}}\right)^{3 / 2}}+\mathcal{O}\left(\epsilon^{2}\right) \\
& =1+\epsilon 0.0273 . .+\mathcal{O}\left(\epsilon^{2}\right)
\end{aligned}
$$

where $\mu_{\infty}=1+d /(2 z)=2(1-\epsilon / 8)+\mathcal{O}\left(\epsilon^{2}\right)$ and the integral has been computed numerically. At variance with $\theta_{0}$ for Model A, non-Markovian corrections appear already at one-loop order, as in the DP and Model C [9, 10]. Compared to its Markovian and Gaussian approximations, the resulting exponent $\theta_{\infty}=2-\epsilon 0.195 . .+\mathcal{O}\left(\epsilon^{2}\right)$ is respectively increased and decreased. Accordingly, $t_{\text {cro }}$ has a finite average $\left\langle t_{\text {cro }}\right\rangle \sim m_{0}^{-\left(1-\theta_{0}\right) / \kappa}$ which diverges with a universal exponent upon reducing $m_{0}$. In the opposite limit $m_{0} \rightarrow \infty$, instead, $\left\langle t_{\text {cro }}\right\rangle$ is finite and determined by $t_{\text {micr }}$ (as for the DP [10]).

It is also instructive, though less relevant for the description of systems other than lattice models, to calculate the persistence probability of a $n$-component vector order parameter $\varphi=\left(\varphi_{1}, \varphi_{2}, . ., \varphi_{n}\right)$ with $n>1$ 
and Model A dynamics (see Eqs. (1) and (2)). When the initial magnetization $m_{0}$ is finite, fluctuations which are parallel $\psi_{\sigma}(x, t)$ and transverse $\psi_{\pi}(x, t)$ (with $O(n-$ 1) symmetry) to the average order parameter $m(t)=$ $\langle\varphi(x, t)\rangle$ are expected to have distinct persistence probabilities, $P_{c}^{\sigma}(t)$ and $P_{c}^{\pi}(t)$, respectively. Taking advantage of the results of Ref. [18] one finds that both of them exhibit a crossover as in Eq. (10) with $n$-dependent exponents $\theta_{0}^{\sigma}(n)=\theta_{0}^{\pi}(n)$ (for $t \ll \tau_{m}$ the $O(n)$ symmetry is restored and the results of Ref. [9] apply) and $\theta_{\infty}^{\sigma, \pi}(n)$ which in the Markovian approximation read $\mu_{\infty}^{\sigma}=1+d /(2 z)$ and $\mu_{\infty}^{\pi}=1-d /(2 z)+2 \beta /(\nu z)$. NonMarkovian corrections contribute already at one loop, yielding $\mathcal{R}^{\sigma}=1+\epsilon(0.115 . .+n 0.131 .) /.(8+n)+\mathcal{O}\left(\epsilon^{2}\right)$ and $\mathcal{R}^{\pi}=1+\epsilon 0.055 . . /(8+n)+\mathcal{O}\left(\epsilon^{2}\right)$ (an expansion of $\mathcal{R}^{\pi}$ around $d=2$ can be obtained from the analysis of Ref. [19]). Interestingly enough, by taking advantage of the results of Ref. [18], $P_{c}^{\pi}(t)$ can be computed exactly for $n \rightarrow \infty$ and $2<d<4$. In the short-time regime $t \ll \tau_{m}$, one recovers $\theta_{0}^{\pi}(n \rightarrow \infty)=(d-2) / 4$, i.e., the same expression as for the case $m_{0}=0$ [8] whereas, for $t \gg \tau_{m}$, one finds $\theta_{\infty}^{\pi}(n \rightarrow \infty)=d / 4$. In both cases there are no non-Markovian corrections and indeed the correlation function of the normalized process $X^{\pi}(t)$ associated to $\tilde{M}_{\pi}(t)=L^{-d} \int \mathrm{d}^{d} x \psi_{\pi}(x, t)$ takes the form (6) with $L(\tilde{t})=\tilde{t}(d-2) / 4[\tilde{t}+d /(d-2)]^{1 / 2}$ and $\tau_{m}=3(d-2) /\left(4 m_{0}^{2}\right)$, allowing also the calculation of the full cross-over function. In passing we mention that a similar crossover occurs also in the spherical model with purely dissipative dynamics: By taking advantage of the results of Ref. [20] we find $\theta_{\infty}=\mu_{\infty}=d / 4+1$ (in agreement with the hyperscaling relation) which differs indeed from $\theta_{0}=\mu_{0}=(d-2) / 4[8]$.

\section{MONTE CARLO SIMULATIONS OF THE TWO-DIMENSIONAL ISING MODEL}

To test the expected scaling behavior (see Eq. (10)), the corresponding crossover as well as our theoretical estimate of $\theta_{\infty}$, we performed Monte Carlo simulations of the two-dimensional Ising model (with spins $s_{i}= \pm 1$ ) on a $L \times L$ lattice with periodic boundary conditions and Glauber dynamics. The system is prepared in a random configuration with $N_{+}$up and $N_{-}$down spins, where $N_{ \pm}=L^{2}\left(1 \pm m_{0}\right) / 2$. At each time step, one site is randomly chosen and the move $s_{i} \mapsto-s_{i}$ is accepted or rejected according to Metropolis rates. One time unit corresponds to $L^{2}$ such time steps. The computation of the persistence probability $P_{c}(t)$ requires the knowledge of the magnetization $M(t)=L^{-2}\left\langle\sum_{i} s_{i}\right\rangle$, which we averaged over 2000 thermal realizations. After the quench to $T_{c}$, each system evolves until $\tilde{M}(t)=L^{-2} \sum_{i} s_{i}-M(t)$ first crosses zero. $P_{c}(t)$ is then measured as the fraction of surviving systems at each time $t$, computed by averaging over $10^{5}$ samples. In Fig. 1 we plot $P_{c}(t)$ for

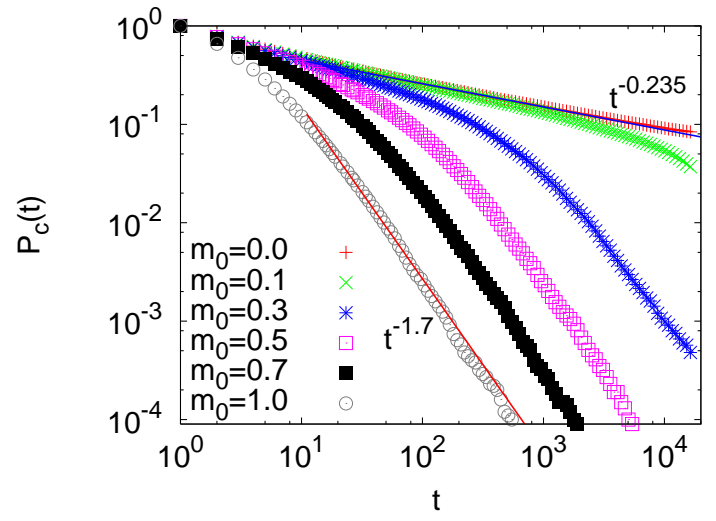

FIG. 1: Persistence probability $P_{c}(t)$ of the two-dimensional critical Ising model with Glauber dynamics, for different initial magnetization $m_{0}$. All the curves decay initially with an exponent $\theta_{0} \simeq 0.235$ and then, after the crossover, with a different exponent $\theta_{\infty} \simeq 1.7$.

$L=256$ and different values of $m_{0}$ ranging from 0 to 1. The curves with $m_{0}>0.1$ display a clear crossover between two different algebraic decays, as anticipated by our theoretical analysis. We have carefully checked by computing $P_{c}(t)$ for $L$ ranging between 64 and $512-$ that the displayed crossover is not affected by finite-size effects. For small enough $t, P_{c}(t)$ decays with an exponent $\theta_{0}^{(\mathrm{MC})}=0.235(5)$ (fully compatible with the available estimate [12]), whereas for larger times the powerlaw decay is faster, with $\theta_{\infty}>\theta_{0}$ and

$$
\theta_{\infty}^{(\mathrm{MC})}=1.7(1) .
$$

An analytical estimate of this exponent can be obtained by setting $\epsilon=2$ in Eq. (12), yielding $\theta_{\infty}^{(1 \mathrm{loop})} \simeq 1.61$ which is actually in rather good agreement with $\theta_{\infty}^{(\mathrm{MC})}$. As expected from the scaling form (10), the time $\tau_{m}$ at which the crossover occurs in Fig. 1increases as the initial magnetization $m_{0}$ decreases and eventually no crossover is observed for $m_{0}=0$ [8]. In Fig. 2 we plot $P_{c}(t) m_{0}^{-\theta_{0} / \kappa}$ $\left(\propto P_{c}(t) \tau_{m}^{\theta_{0}}\right)$ as a function of the rescaled time $t m_{0}^{1 / \kappa}(\propto$ $\left.t / \tau_{m}\right)$ in order to highlight the scaling behavior (10). The exponent $\kappa$ is related to $\beta=1 / 8, \nu=1, \theta^{\prime}=0.191(3)$ 14 and $z=2.1667(5)$ [21] via the scaling relation $\kappa=\theta^{\prime}+$ $\beta /(\nu z)$ [4], which yields $\kappa \simeq 0.249$. For $\theta_{0}$ we use the estimate $\theta_{0}^{(\mathrm{MC})}$ obtained from Fig. 1. The quite good data collapse in Fig. 2 is remarkably obtained without fitting parameters and therefore it provides a convincing numerical evidence of the scaling behavior (10).

\section{CONCLUSIONS}

We have studied the global persistence probability $P_{c}(t)$ for critical system which are initially prepared in a state with short-range correlations and non-vanishing 


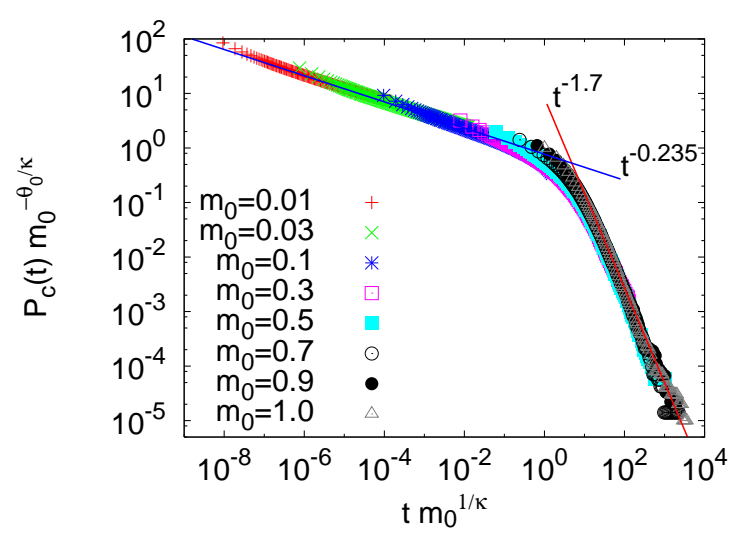

FIG. 2: Plot of $P_{c}(t) m_{0}^{-\theta_{0} / \kappa}$ as a function of the rescaled time $t m_{0}^{1 / \kappa}$ with $\theta_{0}=0.235$ and $\kappa=0.249$, including the data presented in Fig. 1 According to Eq. (10) the exponent of the ultimate power-law decay is $\theta_{\infty} \simeq 1.7$.

average $m_{0}$ of the order parameter. For different models, we have shown that $P_{c}(t)$ exhibits a crossover (apparently overlooked in previous studies) between two distinct power-law behaviors which is described by a universal scaling function $\mathcal{P}$ (see Eq. (10)). Our Monte Carlo simulations of the two-dimensional Ising model with Glauber dynamics clearly display this crossover. We have calculated non-Markovian corrections to the (universal) persistence exponent $\theta_{\infty}$ which characterizes the ultimate long-time behavior of $P_{c}(t)$ for $m_{0}>0$ and it turns out to be in good agreement with our numerical estimates. It would be interesting to extend beyond the Gaussian approximation the calculation of the scaling function $\mathcal{P}$.

In this paper we have studied the crossover in the persistence of the global magnetization $M$ of a $d$-dimensional system at criticality. In addition one might have considered the magnetization $\tilde{M}_{\text {sub }}$ of a $d^{\prime}$-dimensional submanifold of the original system $\left(0 \leq d^{\prime} \leq d\right)$ [22] or the magnetization $M_{V}(t)$ of a subsystem of finite volume $V=\ell^{d}[23]$. It turns out that, depending on $d^{\prime}$, the long-time decay of the persistence probability of $\tilde{M}_{\text {sub }}$ is exponential, stretched exponential, or algebraic [22], whereas the persistence probability of $\tilde{M}_{V}$ is algebraic for $\xi(t) \ll \ell$ and exponential for $\xi(t) \gg \ell$.

The dependence on $d^{\prime}$ and $\ell$ of the dynamic crossover highlighted in this paper and the interplay among the various crossovers surely deserve further investigations.

[1] For a review see L.F. Cugliandolo, Dynamics of glassy systems, in Slow relaxation and nonequilibrium dynamics in condensed matter, J.L. Barrat et al., (Springer-Verlag, Heidelberg, 2003).

[2] L.F. Cugliandolo, J. Kurchan and G. Parisi, J. Phys. I (France) 4, 1641 (1994).

[3] P. Calabrese and A. Gambassi, J. Phys. A 38, R133 (2005).

[4] H.K. Janssen, B. Schaub and B. Schmittmann, Z. Phys. B 73, 539 (1989).

[5] For a review see S.N. Majumdar, Curr. Sci. 77, 370 (1999).

[6] W.Y. Tam, R. Zeitak, K.Y. Szeto and J. Stavans, Phys. Rev. Lett. 78, 1588 (1997); G.P. Wong, M.W. Ross and W. Ronald, Phys. Rev. Lett. 86, 4156 (2001); D.B. Dougherty et al., Phys. Rev. Lett. 89, 136102 (2002).

[7] P.C. Hohenberg and B.I. Halperin, Rev. Mod. Phys. 49, 435 (1977).

[8] S.N. Majumdar, A.J. Bray, S.J. Cornell and C. Sire, Phys. Rev. Lett. 77, 3704 (1996).

[9] K. Oerding, S.J. Cornell and A.J. Bray, Phys. Rev. E 56, R25 (1997).

[10] K. Oerding and F. van Wijland, J. Phys. A 31, 7011 (1998).

[11] S.N. Majumdar and C. Sire, Phys. Rev. Lett. 77, 1420 (1996).

[12] L. Schülke and B. Zheng, Phys. Lett. A 233, 93 (1997).

[13] D. Stauffer, Int. J. Mod. Phys. C 7, 753 (1996).

[14] P. Grassberger, Physica A 214, 547 (1995); Physica A217, 227 (1995)(erratum).

[15] P. Calabrese, A. Gambassi and F. Krzakala, J. Stat. Mech. P06016 (2006).

[16] W. Feller, Introduction to Probability Theory and its Applications, (Vol.2, Wiley, NY, 1996).

[17] D. Slepian, Bell. Syst. Tech. J. 41, 463 (1962).

[18] P. Calabrese and A. Gambassi, J. Stat. Mech. P01001 (2007) [preprint cond-mat/0610266].

[19] A.A. Fedorenko and S. Trimper, Europhys. Lett. 74, 89 (2006).

[20] A. Annibale and P. Sollich, J. Phys. A 39, 2853 (2006).

[21] M.P. Nightingale and H.W.J. Blöte, Phys. Rev. B 62, 1089 (2000).

[22] S.N. Majumdar and A.J. Bray Phys. Rev. Lett. 91, 030602 (2003).

[23] S. Cueille and C. Sire Eur. Phys. J. B 7, 111 (1999).

[24] Note that in reaction-diffusion systems the state with $m_{0}=0$ (and no initial fluctuations - they would anyhow relax exponentially fast in time) correspond to the inactive absorbing phase.

[25] Note that this relation is the same as the one derived in Ref. [9] for the DP universality class. 